\title{
Present Status and Future Prospects of Perovskite Photovoltaics
}

\author{
Solar cells based on metal halide perovskites continue to approach their theoretical \\ performance limits thanks to worldwide research efforts. Mastering the materials \\ properties and addressing stability may allow this technology to bring profound \\ transformations to the electric power generation industry.
}

Metal halide perovskites have fascinated the research community over the last six years, largely due to a combination of their high quality optoelectronic properties - unexpected for materials processed from solution and at low temperature - and the apparent ease with which they can be employed as absorber layers in highly efficient photovoltaic (PV) devices. ${ }^{1}$ Perovskites typically adopt a three dimensional (3D) crystal structure composed of 3 primary ions with a stoichiometry of $\mathrm{ABX}_{3}$. For the all-inorganic compounds, $\mathrm{Cs}$ is the $\mathrm{A}$ cation, and either $\mathrm{Pb}$ or $\mathrm{Sn}$ are the $\mathrm{B}$ cation, with halides of $\mathrm{Cl}, \mathrm{Br}$ or I as the $\mathrm{X}$ anion. The "hybrid" perovskite compounds, which have shown most promise so far, have either methylammonium (MA) or formamidinium (FA) as the A cation. Some of the most efficient and stable compounds even have multiple mixtures of ions at the $A$ and $X$ sites. ${ }^{2-5}$ The rich variety of compositions and the multitude of ways in which they can be investigated have fostered burgeoning interest in perovskites and related compounds.

Since the first reports in 2012, metal halide perovskites were well on their way to deliver high photovoltaic performance ${ }^{1}$, and now single junction cells are already approaching PCE of $23 \%{ }^{6}$ Yet, beyond high efficiency, other critical factors are required for a PV "concept" to transition from the laboratory to real world deployment and use, with ambition to reach TW-scale production capacity. These include low materials and manufacturing costs, high stability, and independence from rare elements. The major components in the perovskite compounds, $\mathrm{Pb}$ and $\mathrm{l}$, are abundant materials, and low cost appears to be preordained. Demonstrating long-term stability, however, has raised major concerns. $^{5}$

In general, there is a sense that the properties which make perovskites such remarkable semiconductors, which are yet to be fully understood, may also be the properties which are more challenging to control for long-term stability. ${ }^{7}$ However, if progress in materials and device engineering will enable long-term PV module deployment, then such improved operational stability, combined with the ease of formation of high quality materials and devices, will place perovskites at the ideal spot to trigger profound transformation in the photovoltaic market.

In this Commentary, the present status in research and directions where further research effort is required will be discussed, but not comprehensively reviewed, along with the status and challenges for technology transfer and commercialisation of perovskite PVs.

\section{Status in research}


Researchers have demonstrated how to routinely obtain perovskite solar cells with efficiency beyond $20 \%$, through changes in materials composition, processing conditions and device architectures. Reaching the maximum theoretical performance in single- and multi-junction solar cells and demonstrating stability over 25 years require further understanding of the optoelectronic properties of metal halide perovskite devices and of how they can be controlled with further chemistry and architecture optimization.

\section{Solar cells with high external radiative efficiency}

Miller, Yablonovitch and Kurtz popularised the notion that a good solar cell must also be a good light emitting diode. ${ }^{8}$ When all non-essential charge recombination paths are eliminated, such as trap assisted recombination at defect or impurity sites, charges present in the active material can only recombine through radiation of light. Thus, bright electroluminescence from a PV cell operating in forward bias (Fig.1a), typically measured when the dark recombination current equals the short-circuit photocurrent under sun light, is a signature that a smaller and smaller fraction of recombination occurs via non-radiative channels. The external radiative efficiency (ERE) of a PV cell, which is the fraction of total dark recombination current which results in the emission of light, is a good indicator of how close to perfection the cell is. Single-digit ERE values, such as 2\% which can be estimated for the record 26.7\% PCE Si cells, ${ }^{9,10}$ are already very good, and the even higher ERE 22\% reached by $28 \%$ PCE GaAs cells ${ }^{9}$ suggests that photon recycling - multiple re-absorption and re-conversion in free charges of photons emitted by charge recombination in the active layer - starts to contribute to both charge transport and voltage gain (Fig.1b). In general, high ERE values correspond to open-circuit voltages $V_{o c}$ approaching the theoretical limits.

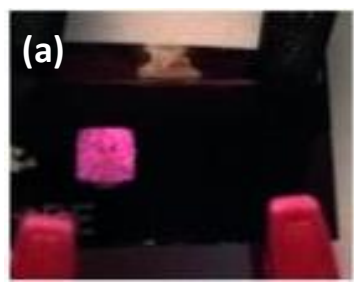

(b)
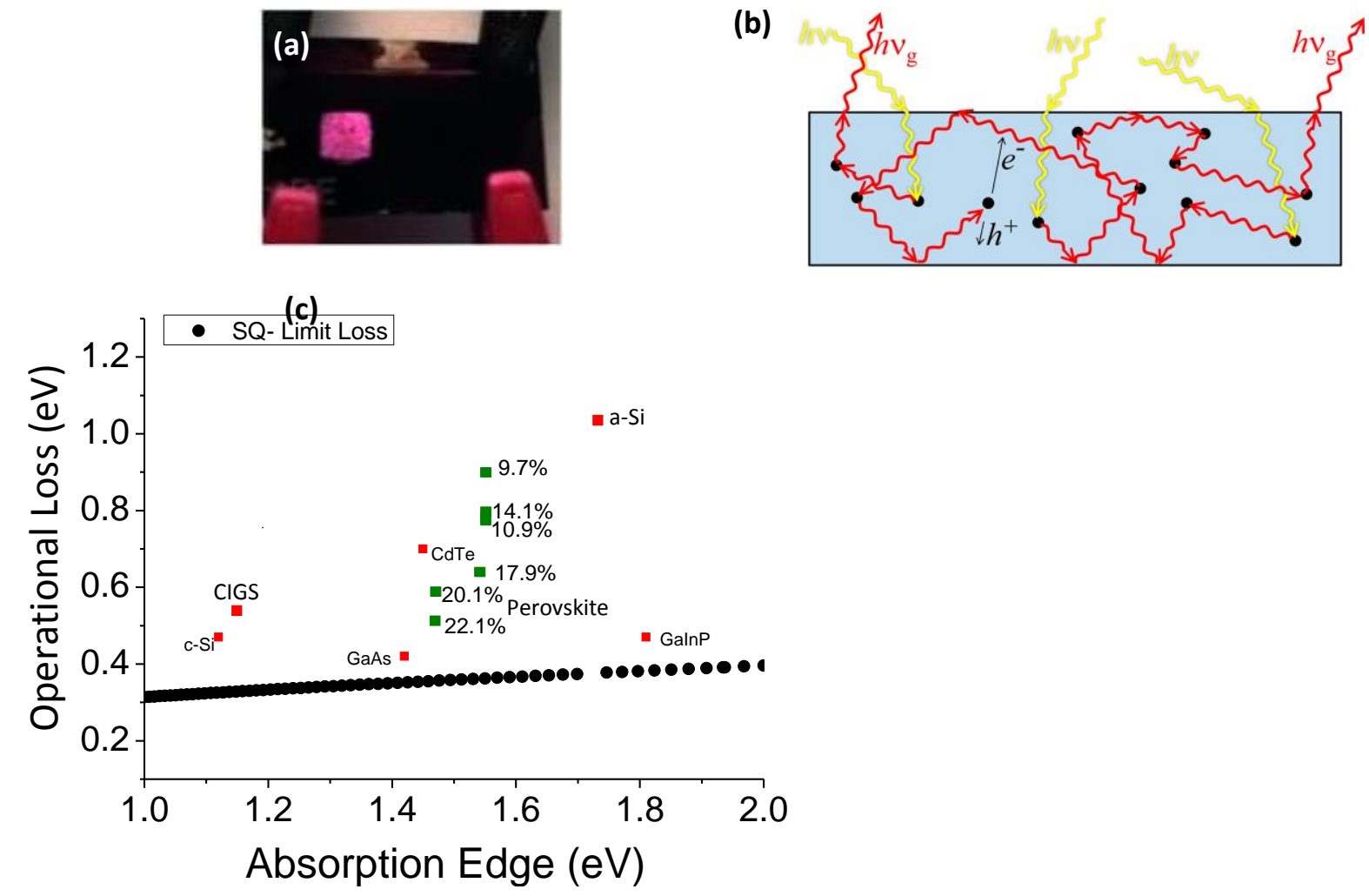
FIGURE1: Light emission and voltage losses in perovskite solar cells. a, Photograph of a perovskite photovoltaic device emitting light, while under forward electrical bias. $\mathbf{b}$, Illustration of photon recycling in a solar absorber layer. External incident light (yellow wavy arrows) generates charge carriers $\left(\mathrm{e}^{-}\right.$and $\mathrm{h}^{+}$), which can recombine (black dots) producing new photons (red wavy arrows) that can either be reabsorbed to generate new charge carriers, or are emitted outside the active layer (light-blue). c, Operational loss versus absorption edge of the solar absorber layer, for a number of different PV technologies (red squares, labelled on figure) and perovskite solar cells (green squares, labels showing the corresponding PCE of the devices). Also shown is the minimum operational loss feasible when a solar cell reaches the Shockley-Queisser (SQ) limit. $\mathbf{a}, \mathbf{b}$ and $\mathbf{c}$ are adapted with permission from references 3,8 and 13 , respectively.

The high luminescence efficiency of metal halide perovskites was recognised early on. ${ }^{11}$ At present, the best perovskite solar cells have an ERE of between 1 to $4 \%,{ }^{3}$ and photon recycling has been suggested to occur in these devices, ${ }^{12}$ - yet their PCE, over $22 \%$, is still lower than that of Si solar cells with comparable ERE. Another closely related indicator of loss in a solar cell is the "operational loss", which is defined as the difference between the optical band gap of the absorber (in eV) and the maximum power point voltage when the cell is operating under sun light. ${ }^{13}$ In Figure $1 c$, the operational loss is shown for a number of different PV technologies, including perovskite solar cells, indicating the reduction in loss as the efficiency has improved. The high ERE and improving operational loss indicate that $\mathrm{V}_{\text {oc }}$ can reach high values; however, the fill factor and the short-circuit current density - the other device parameters determining PCE - are still affected by resistive losses and optical losses, respectively. ${ }^{13}$ Improving the optical design of the cells is important to move towards and beyond $25 \%$ efficiency. It is also noted that the external radiative efficiency of isolated perovskite films, here measured as the fraction of emitted to absorbed light, is as high as $70 \%,{ }^{11}$ thus there is a possibility of achieving much higher EREs in complete solar cells. Importantly, this indicates that when a perovskite absorber layer is sandwiched between charge selective contacts in a photovoltaic device, further recombination losses are introduced. Although various methodologies, such as molecular passivation of the perovskite surface ${ }^{14}$ or reducing the interfacial contact between the charge extraction layers and perovskite absorber ${ }^{15}$, have shown progress in reducing such losses, our present understanding of recombination at these hetero-interfaces is limited, and there remains more than $100 \mathrm{mV}$ to be gained in $\mathrm{V}_{\mathrm{oc}}$, by minimising these losses.

As discussed above, several optoelectronic parameters indicate that the average quality of metal halide perovskites films is already high, which is surprising considering that these are usually deposited through solution processes and have high densities of crystalline defects - this is the reason why these materials have been labelled as defect-tolerant. ${ }^{16,17}$ Yet, it is expected that minimization of electronic defects through materials improvements will be important to progress towards the thermodynamic efficiency limits. An important aspect relates to the chemical processes that occur when depositing perovskite films. While the solutions used obviously contain A, B and X precursors, these ions are incorporated into many forms, such as colloids, dissolved ions, complexes ${ }^{18}$ and the solutions also contain additives, whose role in the chemical reactions and quality of the synthesized perovskites 
needs further understanding. ${ }^{19}$ Often crystallisation proceeds through an intermediate crystalline precursor phase ${ }^{20,21}$ however, there is no consensus yet as to what are the needed "building blocks" to obtain an ideal crystalline perovskite film.

\section{Beyond single-junction solar cells}

A more substantial efficiency boost will be obtained by moving to advanced concepts beyond single-junction PV cells. These may include multi-exciton generation, singlet fission, hot-carrier collection and even intermediate band gap cells. ${ }^{22}$ Results suggesting that some of these processes may occur in perovskite films have been reported, although these have not yet been translated into devices with higher PCE values.

A near term means to move to higher efficiencies is to employ multi-junction concepts, ${ }^{22,23}$ in which perovskite films are combined with silicon or other materials to expand the absorption spectral range - and thus the amount of solar radiation converted into electricity - and convert the solar photons into electrical potential energy at a higher voltage. ${ }^{24,25}$ The success of this approach hinges upon the possibility to tune the optical bandgap of the perovskite layers, ideally from $2 \mathrm{eV}$ to below $1.2 \mathrm{eV}$, depending on the absorption properties of the materials they are combined with. ${ }^{26}$ Although bandgap tuning has been demonstrated by changing the chemical composition or leveraging quantum confinement, at present only the $\sim 1.7$ to $1.5 \mathrm{eV}$ gap cells deliver appropriately high efficiency and stability.

\section{Stability related issues}

These perovskite compounds are largely held together through ionic bonding, and this is likely to be one of the factors central to both the high tolerance to crystalline defects, and the ease at which highly crystalline films can be fabricated at low temperature. ${ }^{27}$

However, this also results in low decomposition temperatures, which make these materials intrinsically less thermally stable than silicon. The use of organic A-site cations, which can withstand much lower temperatures than the inorganic counterparts, is also an intrinsic limit; yet such cations, being larger than any inorganic ion in the periodic table, are necessary to form a stable octahedral framework in the $3 \mathrm{D}$ perovskite structure. ${ }^{28} \mathrm{~A}$ few potential routes have emerged to stabilize these frameworks with inorganic ions, ${ }^{29}$ but more work is required to confirm if these, or other approaches, can lead to long term stable inorganic perovskites.

Aging causes electronic degradation in Silicon arising from the "un-passivation" of dangling bonds on the surface or at grain boundaries. For perovskites, the defect chemistry and physics is still not well understood, but the defects are thought to have energies close to, or inside, the energy bands. ${ }^{16,27}$ Therefore, defects emerging during aging should be less problematic, as suggested by the high efficiency of perovskite solar cells in the presence of a relatively large fraction of $\mathrm{Pbl}_{2}$ (a degradation product) within the film. ${ }^{16}$

Through substitution of ions and improved contact materials, the present generation of perovskite solar cells are vastly more stable, and with appropriate encapsulation may 
already be close to requirements for real world deployment, ${ }^{2,4}$ which are specified in the International Electrotechnical Commission (IEC) 61215 standard. However, in order to make the technology profitable, it is important to have a good level of certainty that the product will last 25 years. This requires going significantly beyond the IEC61215 standard, acquiring more detailed understanding of potential failure modes that could exist.

\section{Materials beyond the $\mathrm{ABX}_{3} \mathrm{~Pb}$-halide perovskites}

Since the advent of efficient metal-halide PV cells, the prospects of discovering new related compounds has redirected new materials discovery. ${ }^{30,31}$ This is also motivated by the desire to replace $\mathrm{Pb}$, due to toxicology concerns. Partial replacement of $\mathrm{Pb}$ with $\mathrm{Sn}$ is presently the only route to reduce the band gap to significantly below $1.5 \mathrm{eV}$, which is essential for multi-junction cells. Although efficient $\mathrm{Sn}$ and $\mathrm{Pb}-\mathrm{Sn} \mathrm{PV}$ cells have been realised, ${ }^{24}$ the stability of the neat $\mathrm{Sn}$ based cells is still extremely low. Other Pb-free compounds have also been tested, however thus far they have not yet delivered PV cells with efficiencies beyond 3\%. ${ }^{31-33}$ This suggests that there is something extraordinarily unique about the Group IV metal halide perovskites. However, it is still likely that new perovskite families do exist, but the challenge of discovering them is much harder than first anticipated.

A separate, successful case is the extension of perovskites to layered structures using larger organic cations. This has resulted in successful realisation of Ruddlesden-Popper phase perovskites, and also films with mixed compositions of 2D and 3D phases. These approaches can result in both improved long-term stability and efficiency. ${ }^{4,34}$ The prospect of creating stable heterojunctions between 2D and 3D phases may also enable both the study of new physics and a better control of perovskite surfaces and electronic interfaces. ${ }^{4,35}$

\section{Technology Transfer in Perovskite PV}

While addressing the open research questions described above will help clarifying the technological potential of perovskite solar cells, there are challenges remaining towards market deployment. Considerations about the availability of materials and their environmental and toxicity risks confirm the role of perovskite PV as strong contenders for large-scale electricity generation. Start-up companies are in the process of delivering the first commercial products, yet more financial support to research and stronger interest from investors are required to overcome barriers to commercialization.

\section{Materials availability and toxicity}

Over the last few years, the cost of mainstream PV power generation has dropped below the cost of producing electricity from coal and gas in the sunniest locations in the world. As efficiency increases further the cost of PV electricity will continue to fall. It is therefore feasible that PV will eventually become our primary source of global power generation, which means that the volume and value of today's PV market is only a fraction of the size it will become over the next few decades. This implies that raw resources must not limit the ambition of any PV technology to scale with this growing market. In this respect, $\mathrm{Pb}$ based 
perovskites sit in a remarkably good position. Fig. 2 shows the current annual global production capacity of elements used in existing PV technologies and perovskites. ${ }^{36}$ In order to reach 12.5 TW PV electricity generation (the dots on the graph), GaAs PV cells would require 500 years of today's Ga production capacity, thin film CdTe PV modules would require 1000 years of today's Te, and thin film CIGS would require 400 years of today's indium. The only commercial PV technology which can scale to this level is silicon, which for 12.5 TW PV electricity generation capacity would require around 3 years of our current $\mathrm{Si}$ production. In stark contrast, perovskites would only take a few days of current $\mathrm{Pb}$ production capacity to scale to the TW level.
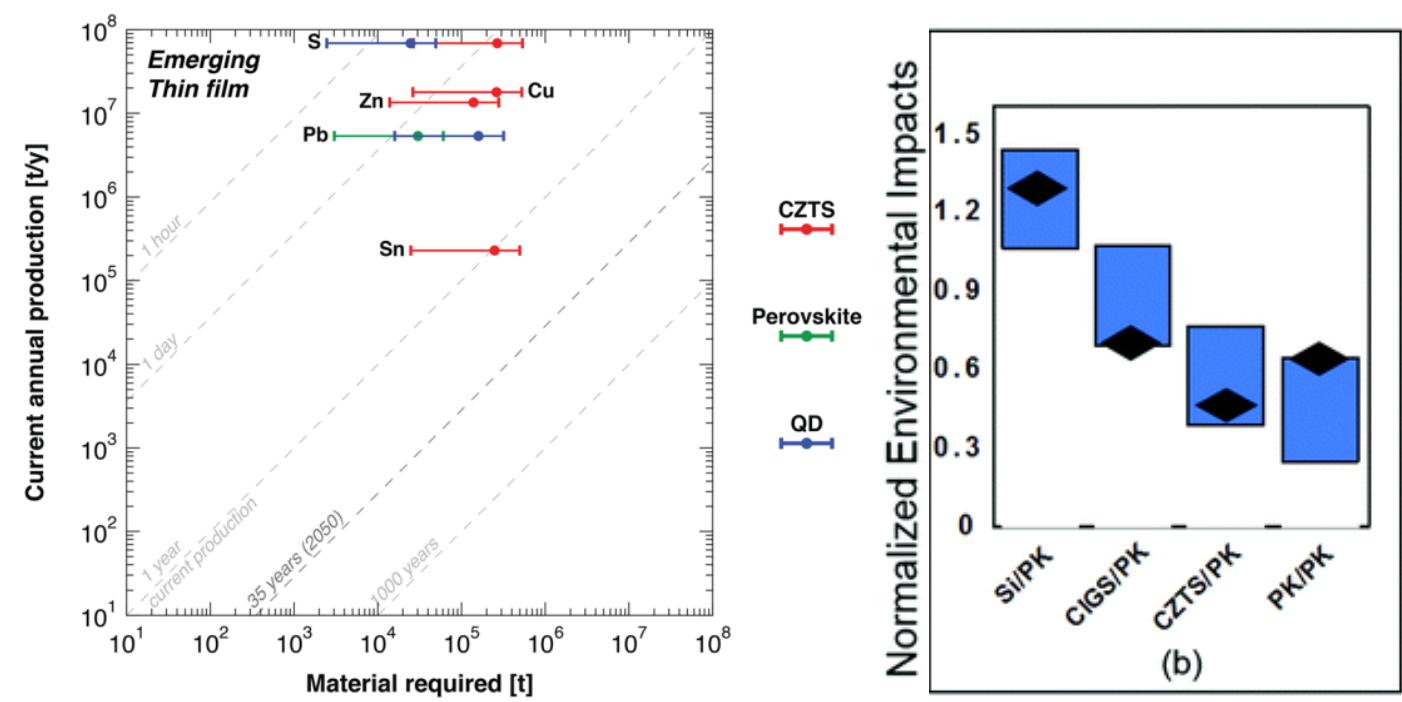

Figure 2. Material Production Capacity and Environmental Impact. a, Annual material production in 2015 , versus material required to deliver $5 \%$ (left end of the bar), $50 \%$ (circle) and $100 \%$ (right end of the bar) of a potential 25TW PV installation for Perovskite, Silicon, GaAs, CIGS and CdTe, adapted with permission from reference. ${ }^{36} \mathbf{b}$, Normalised environmental impact for the manufacturing of different tandem solar cells, where PK = Perovskite, and 1 is the environmental impact for single junction Si-PV production. Adapted with permission from reference 38.

Another critical implication of this analysis is that we would not have to significantly increase our global $\mathrm{Pb}$ production, and hence consumption, beyond to-days levels, if we moved to $100 \% \mathrm{~Pb}$ based perovskite PV power generation, thus containing the environmental impact of $\mathrm{Pb}$-based perovskite PV modules. Yet such impact requires more complete investigation to ensure the sustainability of this technology. As part of the activity within a European project, CHEOPS, SmartGreenScans (http://www.smartgreenscans.nl/\#sthash.ZKpjkplt.MSXJf1t9.dpbs) have undertaken a rigorous life-cycle assessment (LCA) of perovskite-on-silicon tandem cell and module production using the pilot facility in Germany of Oxford Photovoltaics Ltd (https://www.oxfordpv.com/) as a baseline proxy for the manufacturing process. First results show that emitted lead contributes only $0.27 \%$ or less to the total freshwater ecotoxicity and human toxicity. ${ }^{37}$ The majority of the negative environmental or health impacts are related to the monocrystalline silicon production, with Indium also significantly contributing. Similar LCA studies compared the total environmental impact for the manufacturing of different types of perovskite based tandem solar cells. ${ }^{38}$ Consistently, as shown in Fig. $2 b$, 
silicon dominates the total environmental impact, which also encourages the development of perovskite-on-perovskite tandem cells.

\section{Patent Landscape and commercial activity}

As perovskite PV research advances, growing commercial activity is emerging. This is illustrated in a recent review of the patent literature. There are over 2000 filed and 300 granted patents in Perovskite PV. ${ }^{39}$ A chart of the number of patents assigned, per top assignees is shown in Fig.3. Of the top 10 assignees of perovskite patents, 6 are commercial entities, a mixture of start-ups and large corporations, with the remainder being universities or research institutes. Fig. 3a also illustrates the global nature of perovskite PV commercialisation, with top assignees distributed between Europe, the USA, Japan, China, Korea and Australia.

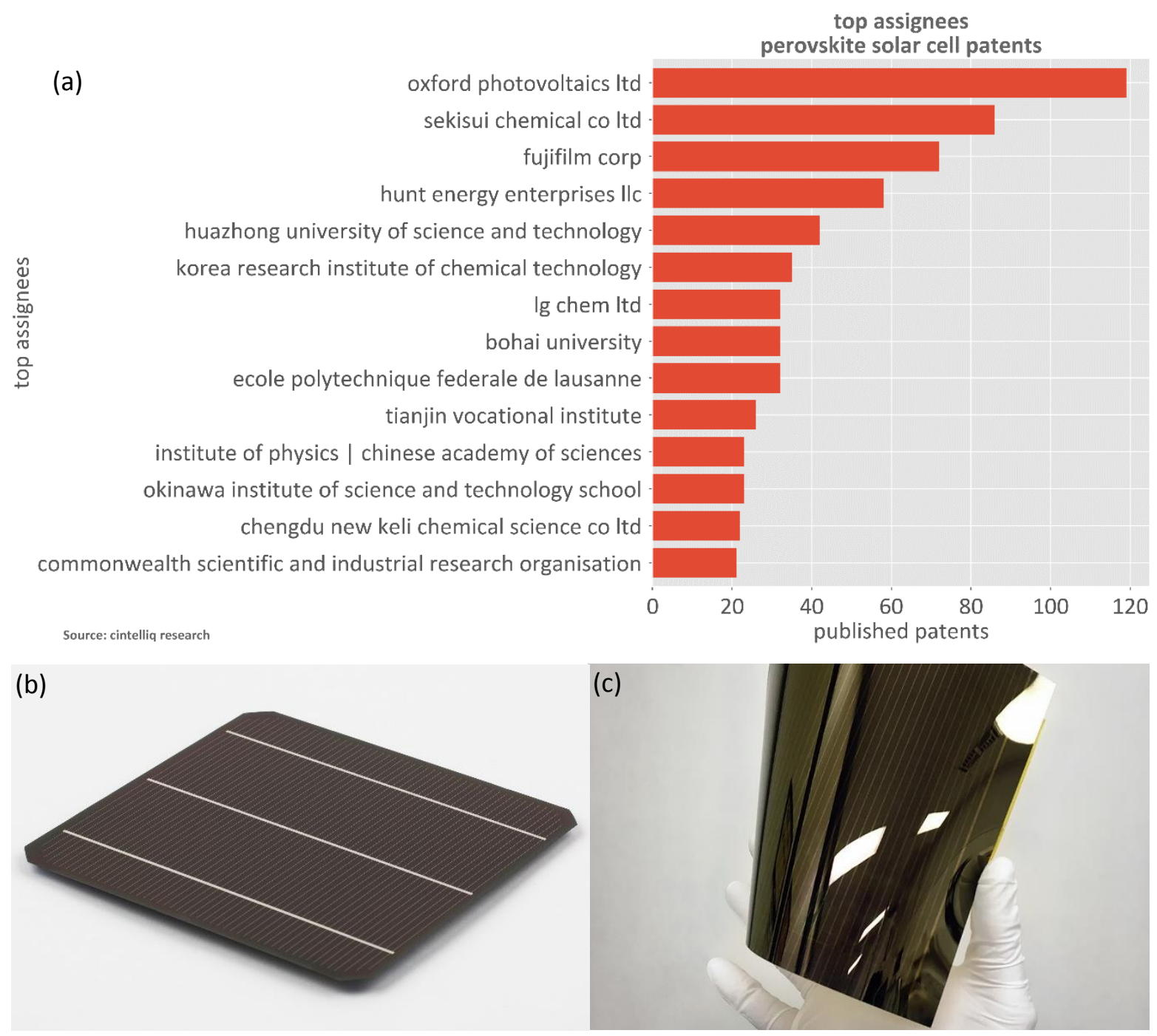

Figure 3. Patents and PV technologies under development. a, List of top 14 assignees of perovskite solar cell patents, reproduced with permission from Cintelliq Research ${ }^{38} \cdot \mathbf{b}, \mathbf{c}$, Photographs of, (b) a $156 \times 156 \mathrm{~mm}$ pseudo-square wafer perovskite-on-silicon tandem cell, courtesy of Oxford 
Photovoltaics Ltd, and (c) a $160 \mathrm{~cm}^{2}$ flexible perovskite module processed via roll-to-roll coating, courtesy of Soliance Solar Research.

There are diverse technical directions being pursued to commercialise perovskite solar technologies, with some companies being more transparent than others. Oxford Photovoltaics Ltd is targeting perovskite-on-silicon tandem solar cells (Fig. $3 \mathrm{~b}$ ). The rationale is that this technology capitalises upon the existing well-developed PV value chain, and through collaborating with existing silicon PV manufacturers, should enable the rapid scaling to large volume manufacturing and address the largest markets. However, there is no hiding from the key requirement to deliver 25 years stability, along with very high efficiency and high yield in manufacturing. Oxford Photovoltaics are presently scaling up towards pilotscale manufacturing in a facility in Germany, which was previously a CIGS pilot production facility. Other companies, such as Saule Technologies (http://sauletech.com), and the Soliance consortium (https://solliance.eu/), which includes Greatcell Solar (www.greatcellsolar.com) and Panasonic (www.panasonic.com), are developing lightweight, flexible and potentially semi-transparent perovskite modules (Fig. 3c), for a broad range of applications including building-integrated PV, the automotive industry, and low light indoor applications.

\section{Support for research and development}

Major initiatives to support perovskite PV research and development are presently lacking in ambition and scale. This may in part be due to the relatively short time passed since perovskite PV has shown such promise, and an inherent inertia and time lag in strategic investment decisions in research. Although over 3000 academic journal publications (in 2017 alone) do suggest that many researchers are working on perovskite PV and the related areas, the funding of this research is through a collection of small grants, rather than large ambitious multilateral projects. Some examples of the larger initiatives in Perovskite PV research, include the US Office of Naval Research (ONR) Air Force Office of Scientific Research (AFOSR) programmes on organic photovoltaics

(https://www.onr.navy.mil/en/Science-Technology/Departments/Code-33/All-Programs/332-navalmaterials/Organic-Photovoltaics), which have refocussed much of their funding towards perovskite based PV, and the US SunShot initiative

(https://www.energy.gov/eere/solar/sunshot-initiative), which funds a number of perovskite PV projects. There is also significant activity in Asia, such as the support provided by the Japanese New Energy and Industrial Technologies Development Organisation (NEDO) (http://www.nedo.go.jp/english/introducing pja.html). In the UK, the total funding of Solar Technologies by the engineering and physical sciences research council (EPSRC) amounts to approximately $£ 32$ million (which is $0.66 \%$ of the EPSRCs portfolio), of which approximately half is related to perovskite solar cell research (https://www.epsrc.ac.uk/research/ourportfolio/researchareas/solartech/). From the European Union, there is presently 1 running, and 4 commencing medium scale collaborative projects focussed on perovskite PV, which amounts to around 25 million euros, in addition to around 20 individual grants from the Marie Curie Fellowship, or European Research Council, amounting to another 10 million euros. If we compare this to 
energy storage, in UK EPSRC provides around $£ 110$ million in funding, including the recently launched Faraday Institution, which accounts for $£ 55$ million. The storage activities are presently mainly focussing on battery storage for the automotive industry, with a f246 million commitment for the Faraday Challenge. On nuclear fusion research, the UK has committed $\mathrm{f} 86$ million for advancing national research (http://www.ccfe.ac.uk/), and on a European scale the investment is two orders of magnitude larger, with the total ITER project (https://www.iter.org/) estimated to cost in excess of 14 billion Euros.

In comparison to scale of the fusion project, solar PV, and specifically perovskites, are receiving less than $1 \%$ of the investment in research and development. However, there exists a tangible prospect for perovskite PV to contribute to the radical transformation of the 100Bn US\$ PV industry and the multi-trillion US\$ global power industry in the near to medium term. The opportunity to capture future value from this industry, by individual countries and multinational initiatives investing in advancing research in perovskite PV, has not yet been realised. Despite the fact that real $1^{\text {st }}$ generation products may enter the market over the next few years, there remains the opportunity for major further innovation over the next few decades.

It is also important to note that government investment into research should not only be made into pre-commercial technologies, but also into technologies which support growing industry. In Europe, research projects supporting silicon PV are unlikely to be funded in the next rounds. However, there remain many prospects to innovate even with this well-established Si technology; by also investing in schemes which make the environment economically favourable for commercialisation, value would be pulled back to the countries which make the investment in research and development.

\section{Barriers to commercialisation}

There are hurdles to overcome on the path to commercial success. The most significant ones are probably not technical, but relate to practicalities of business, where a key issue is raising finance. Scaling up from very promising research results to a technology ready for manufacturing requires an order of magnitude increase in investment. Many venture firms, especially from the USA, remain cautious of investing in PV technology, due to the previous surge in PV investment in the mid 2000's, where the likes of MiaSole, Nanosolar and Solyndra either filed for bankruptcy or sold for a fraction of the investment that had attracted. ${ }^{40}$ However, it is the opinion of the author that investors should be more optimistic today, since the PV industry is in a completely different position as compared to the period between 2008 and 2012. The cost of electricity from PV is now cheaper than any other source in many places in the world, and the downward trend in cost is set to continue over the next decade. Therefore, PV is set to become a major component of global electricity generation and hence the market scale is assured. This makes strategic investment feasible; however, the largest investments in PV at present go into deployment and investment in solar farms, which have predictable rates of return, rather than investments into development of new technology where the risk is much higher. Another non-technical issue, which also effects the ability to raise investment, is how to develop an appropriate business model, which will create value from the PV industry, in the event of 
technical success. Existing mainstream PV manufacturers do not make a reliable profit margin, hence new approaches to creating value, or a change in the structure of the industry, have to emerge.

\section{Outlook}

There has been tremendous research activity in metal halide perovskites for PV applications over the last 6 years, which has resulted in many discoveries and advances. Although expanding upon the lead halide material set has proven challenging, improving the quality and stability of the lead halide perovskites and devices has brought the technology tangibly close to commercial readiness. There is now growing industrial momentum and it is likely that real PV applications employing metal halide perovskites will be demonstrated over the next few years, which will represent a $2^{\text {nd }}$ phase, or era, for this research community.

\section{Acknowledgements}

Henry Snaith's research and time is funded by EPSRC, UK and the European Union's Horizon 2020 framework programme for research and innovation under grant agreement No 653296 of the CHEOPS project.

\section{Affiliations}

Henry Snaith is at the University of Oxford, Department of Physics, Parks Road, Oxford, OX13PU, UK. email: henry.snaith@physics.ox.ac.uk

\section{Competing interests}

Henry Snaith is Co-Founder and Chief Scientific Officer of Oxford Photovoltaics Ltd.

\section{References}

1 Green, M. A., Ho-Baillie, A. \& Snaith, H. J. The emergence of perovskite solar cells. Nature Photonics 8, 506, doi:10.1038/nphoton.2014.134 (2014).

2 Bush, K. A. et al. 23.6\%-efficient monolithic perovskite/silicon tandem solar cells with improved stability. Nature Energy 2, doi:10.1038/nenergy.2017.9 (2017).

3 Saliba, M. et al. Incorporation of rubidium cations into perovskite solar cells improves photovoltaic performance. Science 354, 206-209, doi:10.1126/science.aah5557 (2016).

4 Wang, Z. P. et al. Efficient ambient-air-stable solar cells with 2D-3D heterostructured butylammonium-caesium-formamidinium lead halide perovskites. Nature Energy 2, 17135, doi:10.1038/nenergy.2017.135 (2017).

5 Asghar, M. I., Zhang, J., Wang, H. \& Lund, P. D. Device stability of perovskite solar cells - A review. Renewable and Sustainable Energy Reviews 77, 131-146, doi:https://doi.org/10.1016/j.rser.2017.04.003 (2017).

6 Green, M. A. \& Ho-Baillie, A. Perovskite Solar Cells: The Birth of a New Era in Photovoltaics. ACS Energy Letters 2, 822-830, doi:10.1021/acsenergylett.7b00137 (2017). 

Breaking of Lead Halide Perovskites. Accounts of Chemical Research 49, 330-338, doi:10.1021/acs.accounts.5b00455 (2016). Solar Cells Approach the Shockley\&\#x2013; Queisser Limit. IEEE Journal of Photovoltaics 2, 303-311, doi:10.1109/JPHOTOV.2012.2198434 (2012).

9 Green, M. A. Radiative efficiency of state-of-the-art photovoltaic cells. Progress in Photovoltaics: Research and Applications 20, 472-476, doi:10.1002/pip.1147 (2012). Yoshikawa, K. et al. Silicon heterojunction solar cell with interdigitated back contacts for a photoconversion efficiency over 26\%. Nature Energy 2, 17032, doi:10.1038/nenergy.2017.32 (2017).

11 Deschler, F. et al. High Photoluminescence Efficiency and Optically Pumped Lasing in Solution-Processed Mixed Halide Perovskite Semiconductors. Journal of Physical Chemistry Letters 5, 1421-1426, doi:10.1021/jz5005285 (2014).

12 Pazos-Outón, L. M. et al. Photon recycling in lead iodide perovskite solar cells. Science 351, 1430-1433, doi:10.1126/science.aaf1168 (2016).

13 Nayak, P. K. \& Cahen, D. Updated Assessment of Possibilities and Limits for Solar Cells. Advanced Materials 26, 1622-1628, doi:10.1002/adma.201304620 (2014).

14 Noel, N. K. et al. Enhanced Photoluminescence and Solar Cell Performance via Lewis Base Passivation of Organic Inorganic Lead Halide Perovskites. Acs Nano 8, 9815-9821, doi:10.1021/nn5036476 (2014).

15 Wolff, C. M. et al. Reduced Interface-Mediated Recombination for High Open-Circuit Voltages in CH3NH3Pbl3 Solar Cells. Advanced Materials 29, 1700159-n/a, doi:10.1002/adma.201700159 (2017).

16 Steirer, K. X. et al. Defect Tolerance in Methylammonium Lead Triiodide Perovskite. ACS Energy Letters 1, 360-366, doi:10.1021/acsenergylett.6b00196 (2016).

17 Yin, W.-J., Shi, T. \& Yan, Y. Unusual defect physics in CH3NH3Pbl3 perovskite solar cell absorber. Applied Physics Letters 104, 063903, doi:10.1063/1.4864778 (2014). Yan, K. et al. Hybrid Halide Perovskite Solar Cell Precursors: Colloidal Chemistry and Coordination Engineering behind Device Processing for High Efficiency. Journal of the American Chemical Society 137, 4460-4468, doi:10.1021/jacs.5b00321 (2015).

19 Noel, N. K. et al. Unveiling the Influence of pH on the Crystallization of Hybrid Perovskites, Delivering Low Voltage Loss Photovoltaics. Joule 1, 328-343, doi:10.1016/j.joule.2017.09.009.

20 Moore, D. T. et al. Crystallization Kinetics of Organic-Inorganic Trihalide Perovskites and the Role of the Lead Anion in Crystal Growth. Journal of the American Chemical Society 137, 2350-2358, doi:10.1021/ja512117e (2015).

21 Wang, F., Yu, H., Xu, H. \& Zhao, N. HPbl3: A New Precursor Compound for Highly Efficient Solution-Processed Perovskite Solar Cells. Advanced Functional Materials 25, 1120-1126, doi:10.1002/adfm.201404007 (2015).

22 Green, M. A. \& Bremner, S. P. Energy conversion approaches and materials for highefficiency photovoltaics. Nature Materials 16, 23, doi:10.1038/nmat4676 (2016).

23 Green, M. A. Third generation photovoltaics: solar cells for 2020 and beyond. Physica E: Lowdimensional Systems and Nanostructures 14, 65-70, doi:https://doi.org/10.1016/S13869477(02)00361-2 (2002).

24 Eperon, G. E., Horantner, M. T. \& Snaith, H. J. Metal halide perovskite tandem and multiplejunction photovoltaics. Nature Reviews Chemistry 1, doi:10.1038/s41570-017-0095 (2017).

25 Lal, N. N. et al. Perovskite Tandem Solar Cells. Advanced Energy Materials 7, 1602761-n/a, doi:10.1002/aenm.201602761 (2017).

26 Horantner, M. T. et al. The Potential of Multijunction Perovskite Solar Cells. Acs Energy Letters 2, 2506-2513, doi:10.1021/acsenergylett.7b00647 (2017). 
27 Kim, J., Lee, S.-H., Lee, J. H. \& Hong, K.-H. The Role of Intrinsic Defects in Methylammonium Lead lodide Perovskite. The Journal of Physical Chemistry Letters 5, 1312-1317, doi:10.1021/jz500370k (2014).

28 Filip, M. R., Eperon, G. E., Snaith, H. J. \& Giustino, F. Steric engineering of metal-halide perovskites with tunable optical band gaps. Nature Communications $\mathbf{5}$, doi:10.1038/ncomms6757 (2014).

29 Swarnkar, A. et al. Quantum dot-induced phase stabilization of $\alpha$-CsPbl<sub $>3</$ sub $>$ perovskite for high-efficiency photovoltaics. Science 354, 92-95, doi:10.1126/science.aag2700 (2016).

30 Brandt, R. E. et al. Searching for "Defect-Tolerant" Photovoltaic Materials: Combined Theoretical and Experimental Screening. Chemistry of Materials 29, 4667-4674, doi:10.1021/acs.chemmater.6b05496 (2017).

31 Hoye, R. L. Z. et al. Perovskite-Inspired Photovoltaic Materials: Toward Best Practices in Materials Characterization and Calculations. Chemistry of Materials 29, 1964-1988, doi:10.1021/acs.chemmater.6b03852 (2017).

32 Giustino, F. \& Snaith, H. J. Toward Lead-Free Perovskite Solar Cells. ACS Energy Letters 1, 1233-1240, doi:10.1021/acsenergylett.6b00499 (2016).

33 Greul, E., Petrus, M., Binek, A., Docampo, P. \& Bein, T. Highly stable, phase pure Cs2AgBiBr6 double perovskite thin films for optoelectronic applications. Journal of Materials Chemistry $A$ 5, 19972-19981, doi:10.1039/C7TA06816F (2017).

34 Quan, L. N. et al. Ligand-Stabilized Reduced-Dimensionality Perovskites. Journal of the American Chemical Society 138, 2649-2655, doi:10.1021/jacs.5b11740 (2016).

$35 \quad \mathrm{Hu}$, Y. et al. Hybrid Perovskite/Perovskite Heterojunction Solar Cells. ACS Nano 10, 59996007, doi:10.1021/acsnano.6b01535 (2016).

36 Jean, J., Brown, P. R., Jaffe, R. L., Buonassisi, T. \& Bulovic, V. Pathways for solar photovoltaics. Energy \& Environmental Science 8, 1200-1219, doi:10.1039/C4EE04073B (2015).

37 http://www.cheops-project.eu/news-in-brief/first-results-regarding-the-environmentalimpact-of-perovskitesilicon-tandem-pv-modules, (accessed 14th March 2018).

38 Celik, l. et al. Environmental analysis of perovskites and other relevant solar cell technologies in a tandem configuration. Energy \& Environmental Science 10, 1874-1884, doi:10.1039/C7EEO1650F (2017).

39 https://www.cintelliq.com/research/report/perovskite, (accessed 14th March 2018).

40 https://www.greentechmedia.com/articles/read/rest-in-peace-the-list-of-deceased-solarcompanies\#gs. RimaQt0, (accessed March 14th 2018). 